\title{
Comparative analysis of Voltage Source Inverter with clamper circuit at various modulation indexes
}

\author{
Suchita Siddh ${ }^{1}$, Bharat Modi $^{1}$, Mahendra Lalwani ${ }^{2}$ \\ ${ }^{1}$ Department of Electrical Engineering, Swami Keshvanand Institute of Technology, Management \& \\ Gramothan, Jaipur-302017 (INDIA) \\ ${ }^{2}$ Department of Electrical Engineering, Rajasthan Technical University, Kota, 324001 (INDIA) \\ Email: suchitasiddh@email.com,bharatalone@gmail.com,mlalwani.ee@gmail.com \\ Received 27.11.2020, received in revised form 27.12.2020, accepted 18.01.2021
}

\begin{abstract}
Techniques for pulse width modulation (PWM) have been the focus of several years of research work. Detailed observations made about the effectiveness of the different alternatives. In terms of harmonics based on overall research, the natural and asymmetrical PWM with the third harmonic injection technique gives the best results. Even at different topologies of modulation, these methods have several benefits for higher values of modulation ratio. Here, for generating gate pulses of voltage source inverter, the third harmonic injection-based PWM approach has been used. A controller (which can detect the lower order harmonics reflects in the system output) connected to the inverter. It can also inject the required system harmonics.
\end{abstract}

Keywords- VSI, SPWM, THIPWM, THD Controller/Compensator

\section{INTRODUCTION}

Power electronics has since become a key specialty in electrical engineering. Power electronics use switching mode power semiconductor (SMPS) devices to transform and control electric power in the wide spectrum from $\mathrm{mW}$ to GW. Globally, the use of electrical energy increases exponentially to raise our standard of living. Bimal K. Bose [5] offers a concise but extensive review of recent progress in power electronics and variable frequency drives, including the description of power semiconductor devices, converters, electrical machines, motors, automated control and measurement methods, and simulation methods. Fig. 1 displays a regular two-level three-wire, threephase voltage source inverter with a single-pole double-throw switch with each leg able of blocking uni-polar voltage and running a two-way bidirectional current. Pulse Width Modulation (PWM) is a simple approach of producing the desired output that compares the switched VSI output with the appropriate time-average measurement relation [7]. Sinusoidal PWM (SPWM), as it is easy to establish, is a simple carrier-based PWM method [2]. One main disadvantage of SPWM, however, is the linearity restriction, which limits the linear region up to modulation index $(\mathrm{m})=1$. The ratio of the modulating wave to the carrier wave is given by the modulation index (m) [7]. Due to the generation of low-order harmonics in the output waveform, which has an amplitude of higher value, the operation of VSI on the other side of the linear region is complex. Therefore, the general method to overcome this disadvantage of SPWM is the injection of third-harmonic. Via the use of thirdharmonic injection PWM, the linear area spectrum can be expanded [7]. Because the variation of the harmonic spectrum is influenced by the third harmonic injection, it is therefore chosen to decrease THD in the VSI output. There are major differences in the amplitude of harmonics apart from the third for other transformation in the modulation index or in the frequency ratio. Consequently, in the third harmonic, only small tweaks occur, which indicates that the receptive components are these harmonics. This harmonic response made third harmonic amplitude almost inevitable, so the effect of the above-described harmonics [11] is a rough estimation to either be ignored. X. Mao [4] explained a methodology to reduce switching loss when complying with significant THD criteria implying variable switching frequency strategies (switching schemes with variable switching frequency over a basic interval of time).

As described above, the switching losses in the inverter with SPWM are more, which further leads to a fall in the efficiency of electrical energy output. It also puts too much pressure on the thermal control of the switch [3]. At very high values of $m$, the smoothness of output waveform becomes very good. As the value of $m$ increases, a very high low-order harmonics $\left(3^{\text {rd }}, 5^{\text {th }}, 7^{\text {th }}, 9^{\text {th }}, 11^{\text {th }}\right)$ appear in the inverter output waveform. As a consequence of high value of $\mathrm{m}$, THD reduces. To compensate this, compensator is used in the system because it generates the friendly harmonics. Friendly harmonics (negative harmonics w.r.t. inverter) nullifies the harmonics which generated during OM operation of inverter. The main objective of this paper is to design a compensator in such a way that it can detect the harmonics of lower order in output waveform of the inverter and can also inject the friendly harmonics. 
This paper's description is as followed. A third harmonic injection history is added in section 2 . Section 3 describes, apart from the previously defined injection schemes, the process used. The simulation model and findings are also discussed in Section 4 and, respectively, the paper is concluded in Section 5.

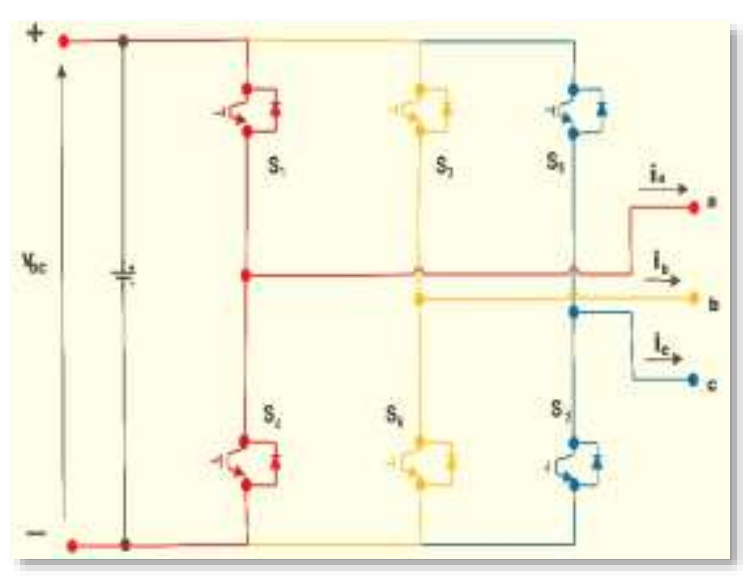

Fig. 1: 2-level 3-wire 3-phse VSI

\section{THIRD HARMONIC INJECTION}

Square wave modulation (which is also called as six-stepped wave) has a very easy design to implement gate pulses to operate VSIs more with highest possible use of DC buses. However this technique contributes to greater THD values and major magnitude of low-order harmonics [6], in response to the uncontrolled fundamental part. In order to minimize these drawbacks, the distribution of PWM waveforms is widely used in two particular ways [7]: perhaps by combining a regular triangular shaped carrier signal with modulating waveform, such as SPWM and THIPWM, or by using a space vector process.

Sideband harmonics arise mostly at and around the frequency of the carrier signal and its component multiples while using SPWM [9]. When its PWM approach is synchronized and the carrier frequency is constant, then perhaps the sidebands will have two centered, salient harmonics. Three sinusoidal patterned modulating signals are used when beginning with SPWM. These references to voltage are fairly well understood in (1) [5]:

$$
\begin{gathered}
V_{A}(t)=V_{p} \sin \left(\omega_{0} t\right) \\
V_{B}(t)=V_{p} \sin \left(\omega_{0} t-\frac{2 \pi}{3}\right) \\
V_{C}(t)=V_{p} \sin \left(\omega_{0} t+\frac{2 \pi}{3}\right)
\end{gathered}
$$

Here, $V_{p}$ is the maximum amplitude of the sine wave, $\omega_{0}$ is the angular frequency whose value is given by $\omega_{0}=2 \pi f_{0}$; and $f_{0}$ is described as the fundamental frequency component.

Depending on the relative values of the carrier wave reference inputs, three-phase voltage source inverters have $2^{3}$ alternating operating states. Its first and the last regions are known as those of the zero states" or relative values $\left(V_{A}, V_{B}\right.$ and, $\left.V_{C}\right)$ depend on the intermediary states. The linear region of SPWM is restricted to modulation index $(m)=1$; any boost over one would move the cycle into a region of operation that is not linear or overmodulation. Operating in the zone of overmodulation has certain advantages, such as achieving a higher fundamental component of voltages without rising the voltage of the DC bus. However, it is handled from generating sub-carrier frequency harmonics and a drop in the value of voltage gain of VSI at the expense of power efficiency, along with causing gate signals to drop [8].

The third harmonic injection [7], which fixes to some extent the problem of linearity, would be utilized to enhance the linear field. In addition, insertion of a third harmonic component affects the sideband components without interrupting the particular fundamental component [10]. The device becomes over-modulated and the pulse drop takes place until the magnitude of the reference signal reaches the same carrier or, approximately equal, when operating with a value of modulation index which will be greater than one. A third harmonic signal is injected in the system to quality structure the peak level and permit the modulating signal to be properly integrated within the range of carrier peaks. This enables output voltages to be produced while being in the linear region. This expansion relies on the magnitude of the third harmonic which is inserted into the sinusoidal reference signal inside the linear field. By adding third harmonic, a set of different correlations is established to build new references is defined in the equation (2) [1].

$$
\begin{gathered}
V_{A}(t)=V_{p} \sin \left(\omega_{0} t\right)+V_{p 3} \sin \left(3 \omega_{0} t\right) \\
V_{B}(t)=V_{p} \sin \left(\omega_{0} t-\frac{2 \pi}{3}\right) \\
\quad+V_{p 3} \sin \left(3 \omega_{0} t-\frac{2 \pi}{3}\right) \\
V_{C}(t)=V_{p} \sin \left(\omega_{0} t+\frac{2 \pi}{3}\right) \\
+V_{p 3} \sin \left(3 \omega_{0} t+\frac{2 \pi}{3}\right)
\end{gathered}
$$

In fact, in all transitions of the phase, the third harmonic components are in-phase. As a result, they balance each other out absolutely. In a line-to-line harmonic system, this study can be extended to all triplen harmonics. Implicitly, the impact on the harmonic spectrum of the third harmonic insertion is irreversible, and it is used to adjust the output waveform's THD. This can be observed by noting that the reference signal inside the carrier peaks can be limited by many injections. Through injection, however, results in a specific content of THD. 


\section{PROPOSED SCHEME}

There are various PWM schemes that were developed in past years, but out of all those schemes, the best-suited scheme for this research work is the third-harmonic injection PWM scheme. The reason behind opting for this scheme is that it provides ease of operating the inverter up to $\mathrm{m}=1.15$; without the occurrence of pulse-dropping.

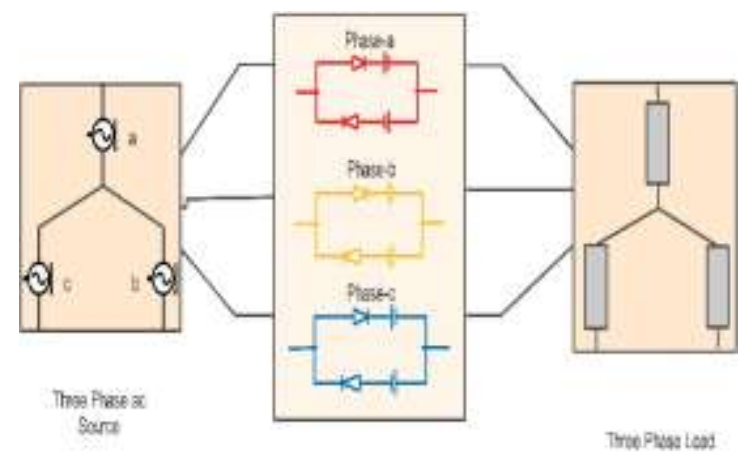

Fig.2 Clipping Circuit model with DC source (Battery)

A basic clipping circuit is shown in figure 2, also call it a controller, has designed here which further connected with the two-level VSI as shown in figure 3. This controller is a combination of a clipping circuit along with the DC supply.

The main purpose of having a controller in this proposed scheme is that it can provide some harmonics which are required for this research work and also it can detect harmonics of the lower order which appears in the system. Another purpose of using controller is that whenever there will be an occurrence of mismatch in the output of either inverter or grid supply, the controller can utilize the maximum value of DC bus voltage.

The activity of switches $S_{1}$ through $S_{6}$ is defined when compared the both i.e. modulating wave and carrier wave. The upper switch $S_{1}$ of leg A in the inverter will be switched on when $V_{p A}$ deflects $V_{c r}$. The lower switch $S_{4}$ works in a complementary way that of the upper switch $S_{1}$ and, is therefore turned off. The corresponding inverter terminal voltage $V_{A N}$ is equal to $V_{d c}$, which is the voltage at the phase A terminal concerning the negative terminal of dc bus. If $V_{p A}<V_{c r}, S_{4}$ is on and $S_{1}$ is off, this leads to $V_{A N}=0$. The inverter is termed as a twolevel inverter since the $V_{A N}$ waveform has only two levels, $V_{d c}$ and 0 . It is possible to independently monitor the magnitude and frequency of $V_{A B 1}$ through $m$ (modulation index) and $f_{m}$ (modulating wave frequency), respectively.

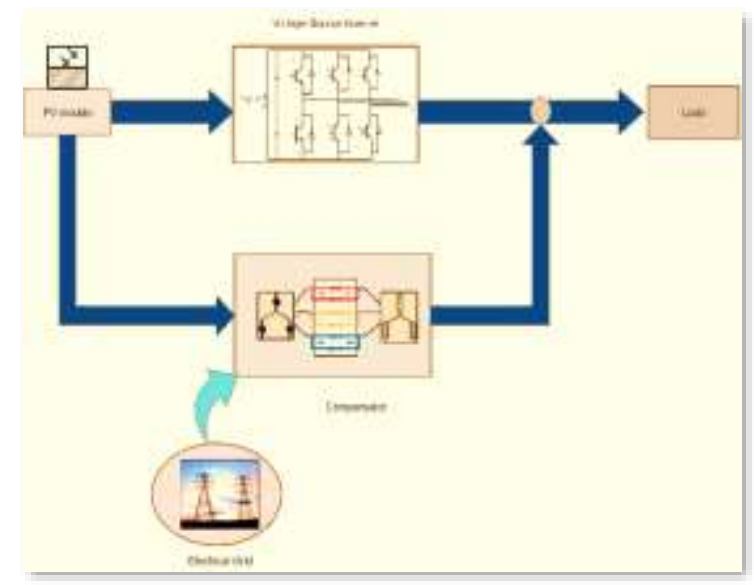

Fig.3 Block diagram of the Proposed Scheme

The fundamental component of inverter voltage $V_{A B 1}$ can also be increased without experiencing over-modulation by simply injecting third harmonic element to three-phase sinusoidal modulating wave. This modulation scheme is called a Third-harmonic injection PWM technique (THIPWM). In the inverter output voltages, the SVM schemes produce both odd- and even-order harmonics. Even order harmonics could not have a major effect on the performance of the motor. The modulating wave $V_{p A}$ consists of a fundamental component $V_{p 1}$ and a component of third harmonics $V_{p 3}$, which renders $V_{p A}$ slightly flattened on top. Here, $V_{p A}$ is the peak value of the modulating wave of phase A. As a result, the fundamental peak $V_{p 1}$ may be larger in comparison with the triangular shaped peak carrier wave $V_{c r}$, which increases the fundamental component of line-to-line voltage $V_{A B 1}$. While, the $V_{p A}$, peak modulating wave can be held lower than $V_{c r}$, preventing overmodulation-related problems. A third harmonic element $V_{p 3}$ injected will not boost $V_{A B}$ 's harmonic distortion. In $V_{A B}$, which is the lineto-line voltage, the third-order harmonic voltage doesn't somehow occur, but it occurs in any of the $V_{A N}, V_{B N}$ and $V_{C N}$, which are basically the value of terminal voltage. This is due to line-to-line voltage which can be defined by -

$$
V_{A B}=V_{A N}-V_{B N}
$$

in which the third-order harmonics in $V_{A N}$ and $V_{B N}$ are mostly of zero sequence and negate each other with the same phase and amplitude displacement. 

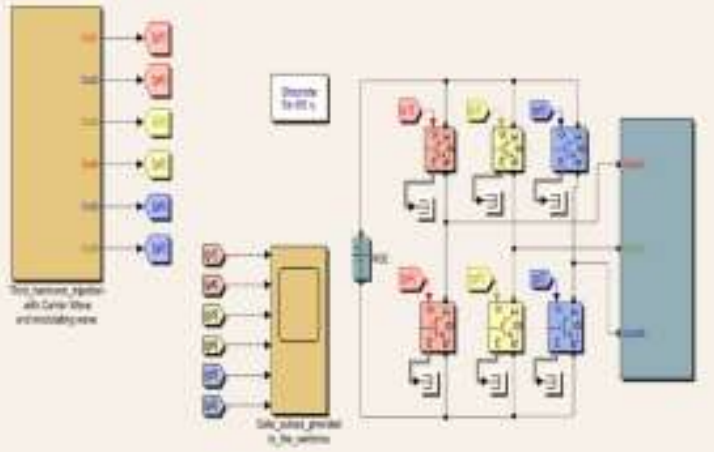

Fig.4 Simulation Model including Subsystem of Controller and Switching Pulses

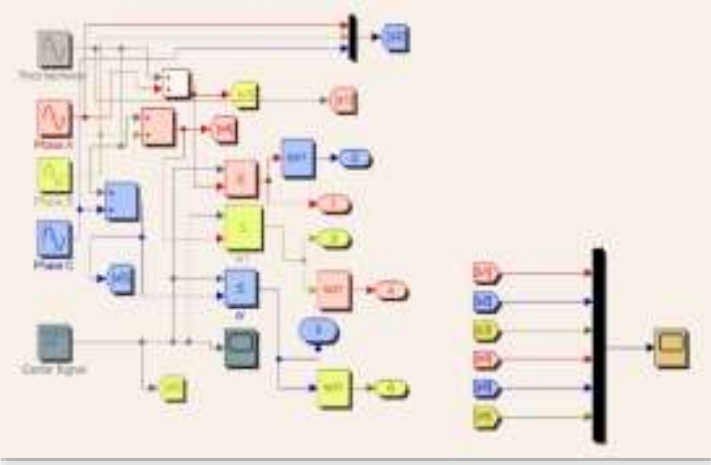

Fig.5 Simulation Model of Switching Pulse Subsystem

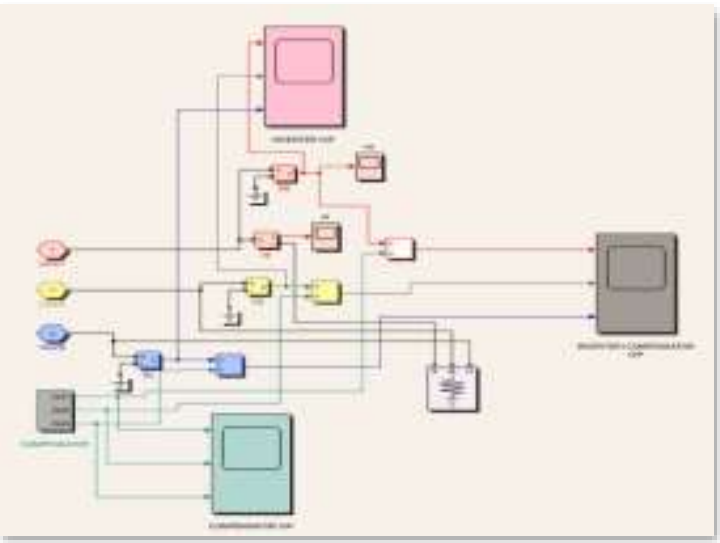

Fig.6 Simulation Model of combining Inverter and Controller Output

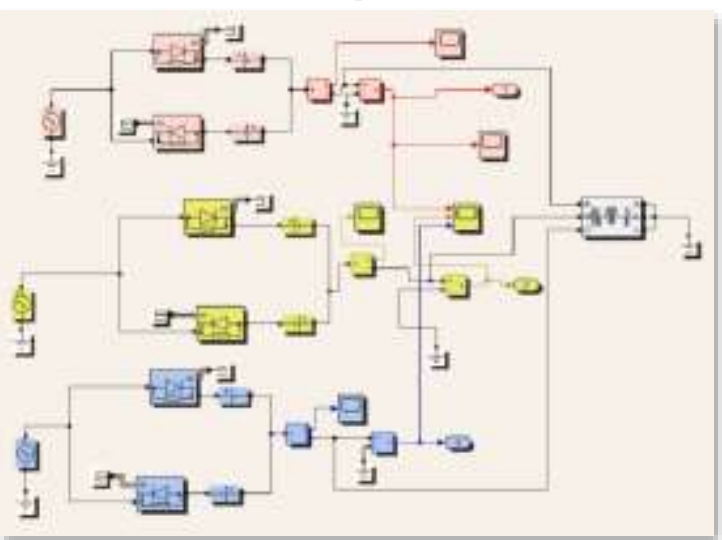

Fig.7 Simulation Model of Controller Subsystem

\section{SIMULATION MODEL AND RESULTS}

Figure 7 shows the simulation model of a three phase controller circuit in which anti-parallel diodes are connected with battery (DC source). Figure 5 shows the injection of third harmonics in the reference modulating signal and carrier pulses. In figure 6 , the outcome of controller circuit and the outcome of inverter has combined for the overall outcome and after that it get checked for different values of modulation index $(m)$.

Figures 11, 15 and, 19 shows the overall outcome (i.e. combined outcome of inverter with controller) at various values of modulation index $(m)$. The controller produces some harmonics because of which the unwanted harmonics which produced in the inverter output, gets nullified. Because of which controller is able to compensate the adverse effects of pulse dropping.

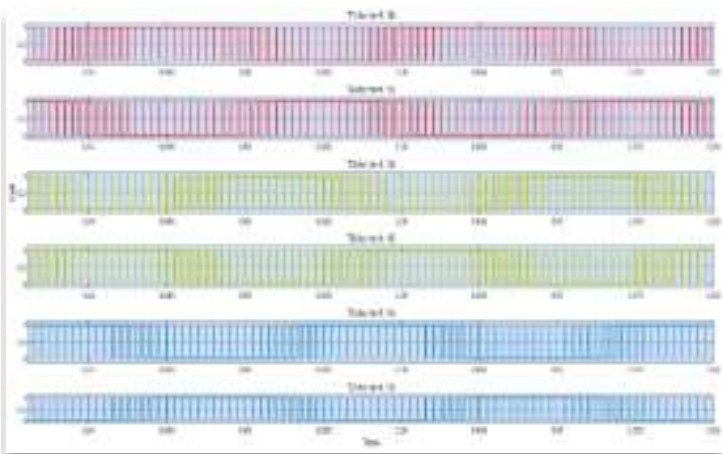

Fig.8 Switching Pulses For Phase (a, b, c) at $\mathrm{m}=1.15$

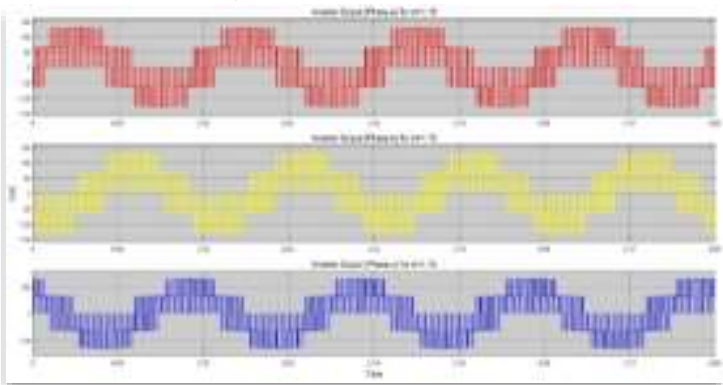

Fig.9 Inverter Output at $m=1.15$

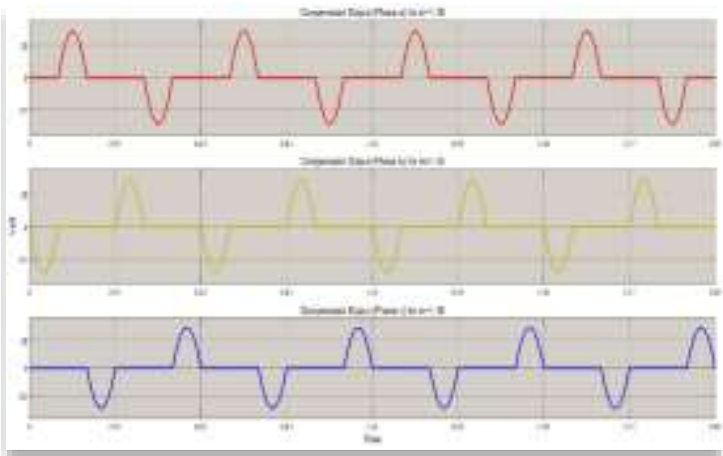

Fig.10 Output of all the phases of the Controller at $\mathrm{m}=1.15$ 


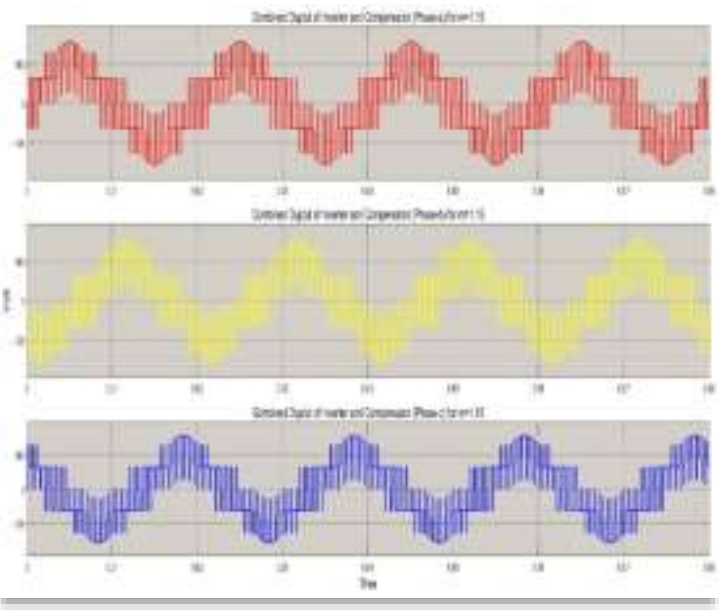

Fig.11 Overall Output at $\mathrm{m}=1.15$

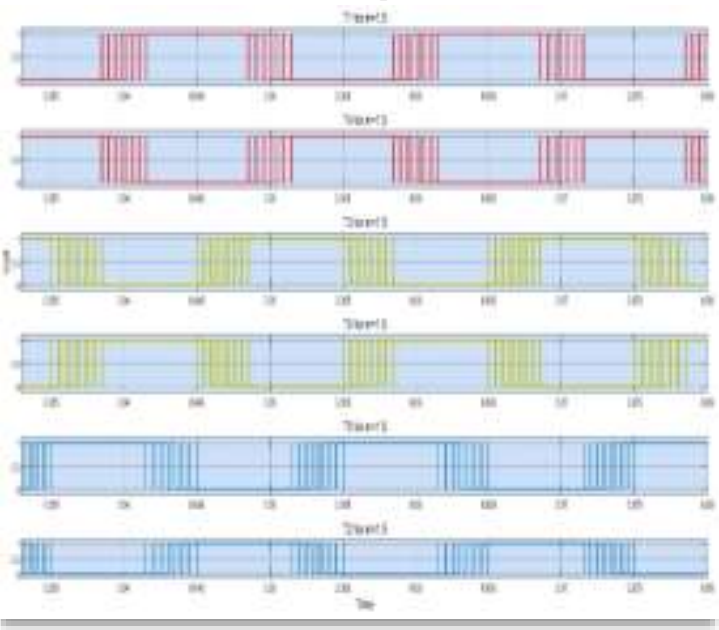

Fig.12 Switching Pulses For Phase (a, b, c) at $\mathrm{m}=1.5$
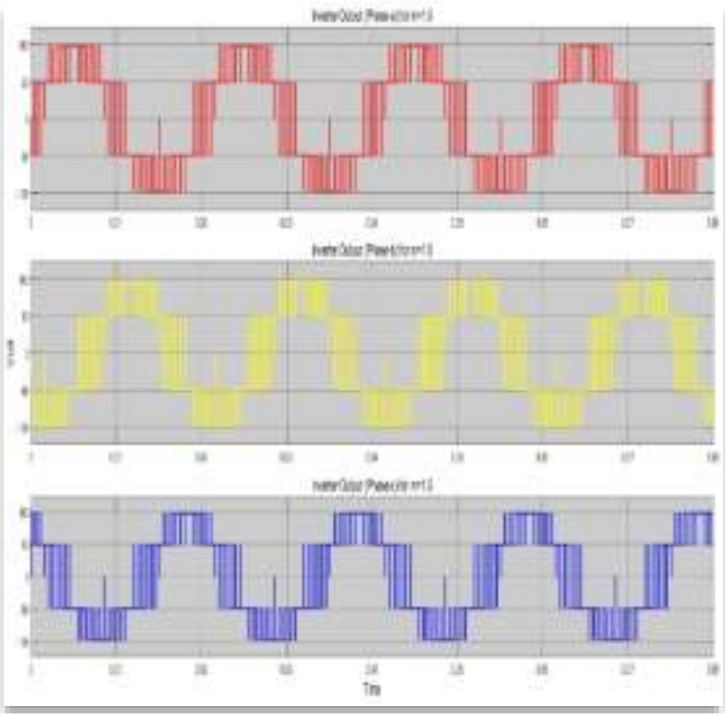

Fig.13 Inverter Output at $m=1.5$

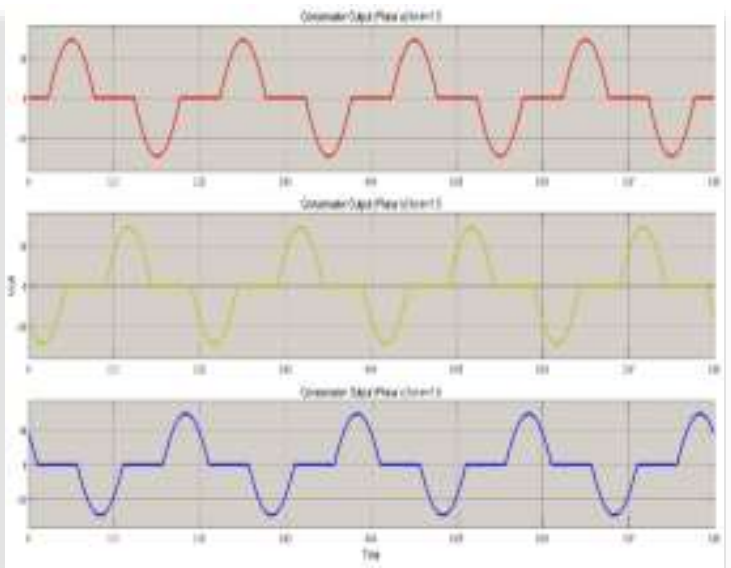

Fig.14 Output of all the phases of the Controller at $m=1.5$

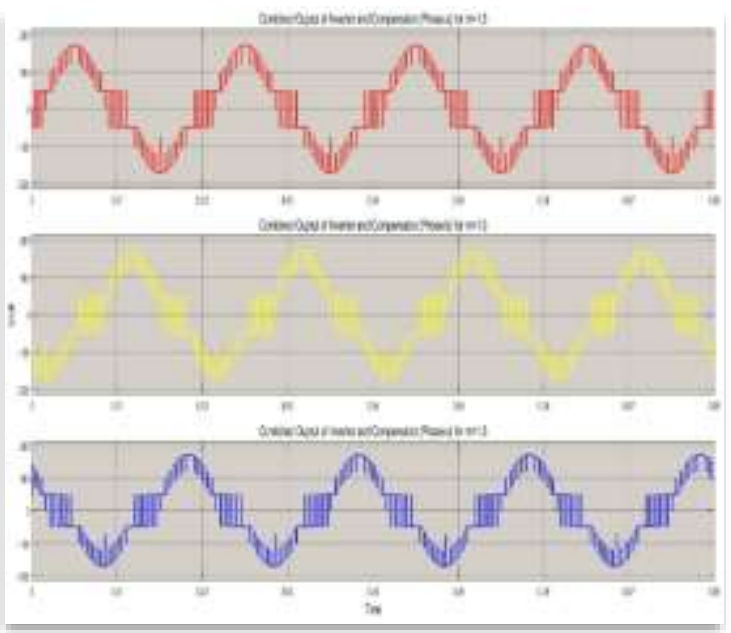

Fig.15 Overall Output at $m=1.5$

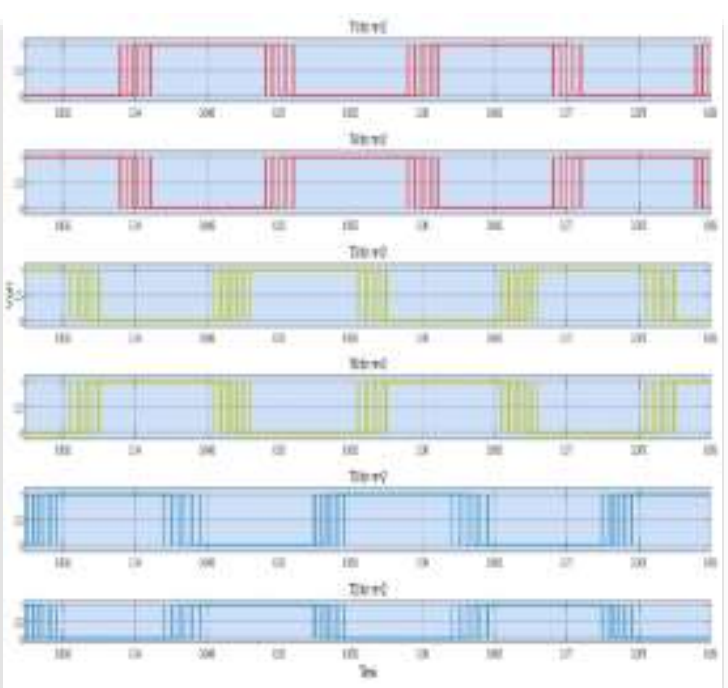

Fig.16 Switching Pulses For Phase (a, b, c) at m=2 


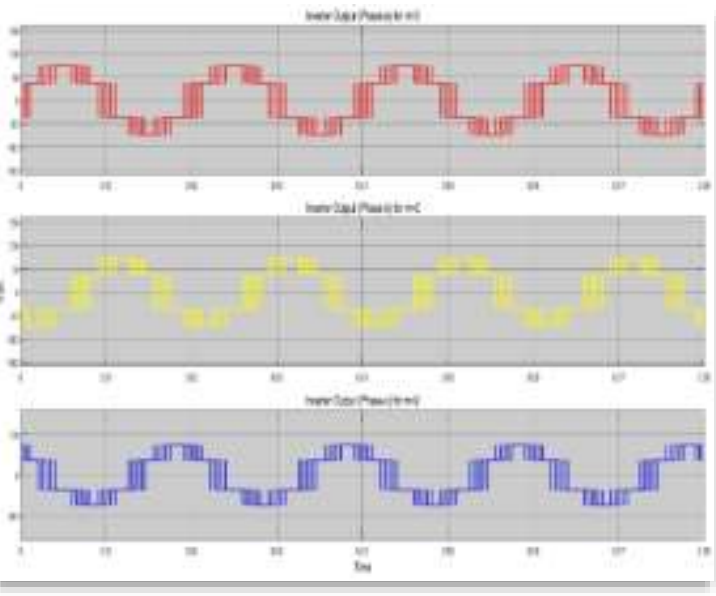

Fig.17 Inverter Output at $m=2$

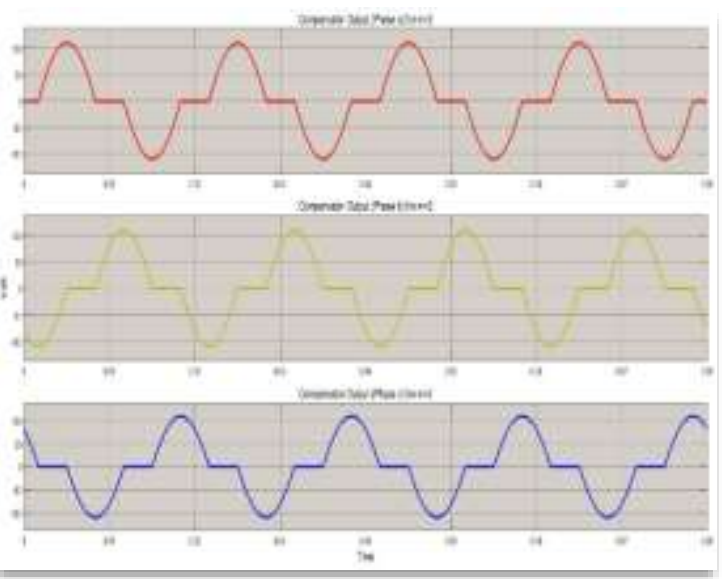

Fig.18 Output of all the phases of the Controller at $m=2$

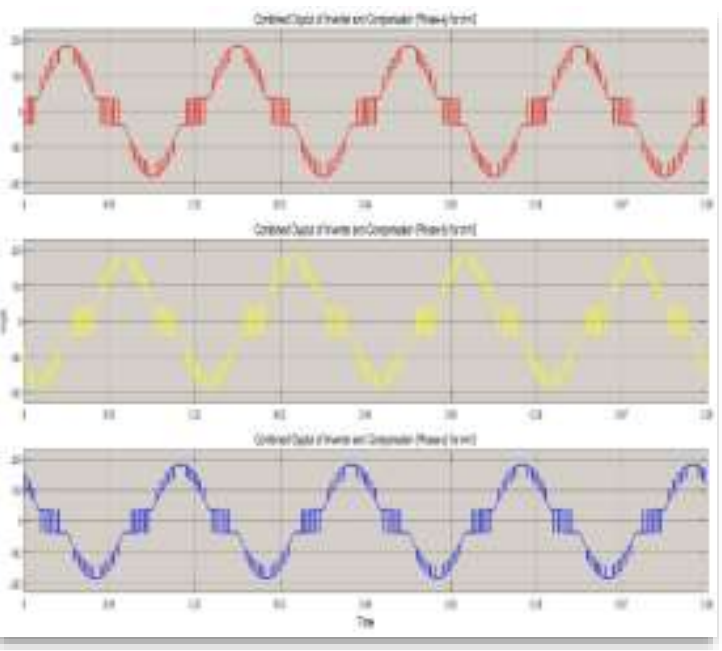

Fig.19 Overall Output at $m=2$

It is clearly seen in figure 8 that when the value of $m$ is taken as 1.15 , the pulse dropping does not take place, whereas when the value of $m$ is choosen as 1.5, the pulse dropping took place as shown in figure 12 and same phenomena took place when the value of $m$ is considered as 2, clearly seen in figure 16. As described previously, this pulse dropping can not able to affect the overall output of the system only because of the controller circuit.

\section{CONCLUSION}

By observing the waveforms of all the phases in figures 11,15 and, 19 , which are the combined waveform of inverter with controller, it seems clearly that the value of THD is very less. However, these waveforms shows the line-toneutral voltage but if we measure the line-to-line voltage than the content of third harmonic is absent as well as the multiple of third harmonic are also absent, therefore, the overall THD value reduces. It is also seen that the injection of third harmonic PWM is efficient for $m=1.15$ and for the above range of $m$, the controller produces some harmonics because of which the unwanted harmonics which produced in the inverter output, gets nullified, therefore, the overall output remains unaffected. This controller is very useful with nonuniform insolation perspective in reference with solar radiation system. An added benefit of such a controller has been that it offers accessibility and automatic feedback capabilities.

\section{REFERENCES}

[1] Albatran, Saher, Abdel Rahman Al Khalaileh, and Ahmad Allabadi. "Minimizing Total Harmonic Distortion of a Two-Level Voltage Source Inverter Using Optimal Third Harmonic Injection." IEEE Transactions on Power Electronics (2019).

[2] Chang, En-Chih, et al. "High-Performance DC-AC Power Converter Using Adaptive Control Technique for Advanced Material Machining." Journal of Automation and Control Engineering Vol 5.1 (2017).

[3] Reichl, John, et al. "Design optimization of hybrid-switch soft-switching inverters using multiscale electrothermal simulation." IEEE Transactions on Power Electronics 32.1 (2016): 503-514

[4] Mao, Xiaolin, Rajapandian Ayyanar, and Harish K. Krishnamurthy. "Optimal variable switching frequency scheme for reducing switching loss in single-phase inverters based on time-domain ripple analysis." IEEE Transactions on Power Electronics 24.4 (2009): 991-1001.

[5] Bose, Bimal K. "Power electronics and motor drives recent progress and perspective." IEEE Transactions on Industrial Electronics 56.2 (2008): 581-588

[6] Mohan, Ned, and Robbins-Power Electronic Converters "Applications and design." (2007).

[7] Holmes, D. Grahame, and Thomas A. Lipo. Pulse width modulation for power converters: principles and practice. Vol. 18. John Wiley \& Sons, 2003.

[8] Hava, Ahmet M., Russel J. Kerkman, and Thomas A. Lipo. "Carrier-based PWM-VSI overmodulation strategies: analysis, comparison, and design." IEEE Transactions on Power Electronics 13.4 (1998): 674-689.

[9] Holtz, Joachim. "Pulsewidth modulation-a survey." PESC'92 Record. 23rd Annual IEEE Power Electronics Specialists Conference. IEEE, 1992.

[10] Theocharis, J., and V. Petridis. "Harmonic insertion in PWM inverter drive schemes." European Transactions on Electrical Power 2.3 (1992): 143-151.

[11] Halász, S., S. R. Bowes, and A. Midoun. "Suboptimal switching strategies for microprocessor-controlled PWM inverter drives." IEE Proceedings B-Electric Power Applications. Vol. 132. No. 6. IET, 1985. 